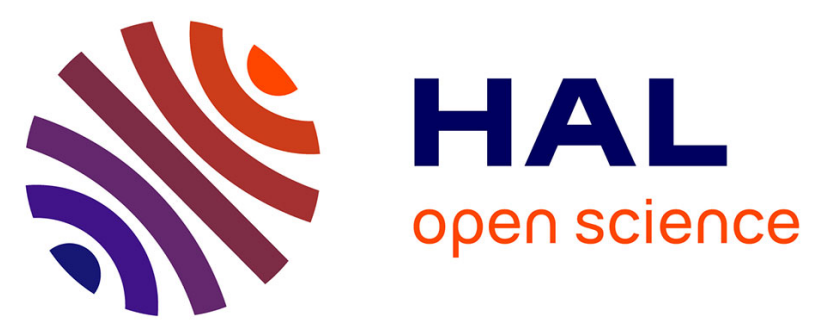

\title{
Treatment with chenodeoxycholic acid in cerebrotendinous xanthomatosis: clinical, neurophysiological, and quantitative brain structural outcomes
}

Maria del Mar Amador, Marion Masingue, Rabab Debs, Foudil Lamari, Vincent Perlbarg, Emmanuel Roze, Bertrand Degos, Fanny Mochel

\section{To cite this version:}

Maria del Mar Amador, Marion Masingue, Rabab Debs, Foudil Lamari, Vincent Perlbarg, et al.. Treatment with chenodeoxycholic acid in cerebrotendinous xanthomatosis: clinical, neurophysiological, and quantitative brain structural outcomes. Journal of Inherited Metabolic Disease, 2018, 41 (5), pp.799-807. 10.1007/s10545-018-0162-7 . hal-01973264

\section{HAL Id: hal-01973264 \\ https://hal.sorbonne-universite.fr/hal-01973264}

Submitted on 8 Jan 2019

HAL is a multi-disciplinary open access archive for the deposit and dissemination of scientific research documents, whether they are published or not. The documents may come from teaching and research institutions in France or abroad, or from public or private research centers.
L'archive ouverte pluridisciplinaire HAL, est destinée au dépôt et à la diffusion de documents scientifiques de niveau recherche, publiés ou non, émanant des établissements d'enseignement et de recherche français ou étrangers, des laboratoires publics ou privés. 


\title{
Treatment with chenodeoxycholic acid in cerebrotendinous xanthomatosis: clinical, neurophysiological, and quantitative brain structural outcomes
}

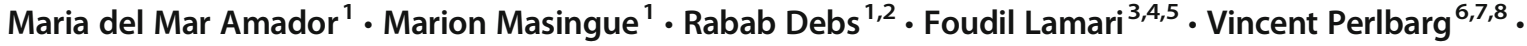 \\ Emmanuel Roze ${ }^{1,4,6} \cdot$ Bertrand Degos $^{9,10} \cdot$ Fanny Mochel $^{4,5,6,11,12}$ (D)
}

\begin{abstract}
Background Cerebrotendinous xanthomatosis (CTX) is a rare neurodegenerative disease related to sterols metabolism. It affects both central and peripheral nervous systems but treatment with chenodeoxycholic acid (CDCA) has been reported to stabilize clinical scores and improve nerve conduction parameters. Few quantitative brain structural studies have been conducted to assess the effect of CDCA in CTX.

Methods and results We collected retrospectively clinical, neurophysiological, and quantitative brain structural data in a cohort of 14 patients with CTX treated by CDCA over a mean period of 5 years. Plasma cholestanol levels normalized under treatment with CDCA within a few months. We observed a significant clinical improvement in patients up to 25 years old, whose treatment was initiated less than 15 years after the onset of neurological symptoms. Conversely, patients whose treatment was initiated more than 25 years after neurological disease onset continued their clinical deterioration. Eleven patients presented with a length-dependent peripheral neuropathy, whose electrophysiological parameters improved significantly under CDCA. Volumetric analyses in a subset of patients showed no overt volume loss under CDCA. Moreover, diffusion weighted imaging showed improved fiber integrity of the ponto-cerebellar and the internal capsule with CDCA. CDCA was well tolerated in all patients with CTX.
\end{abstract}

Maria del Mar Amador, Marion Masingue, and Rabab Debs contributed equally to this work.

Electronic supplementary material The online version of this article (https://doi.org/10.1007/s10545-018-0162-7) contains supplementary material, which is available to authorized users.

Fanny Mochel

fanny.mochel@upmc.fr

1 Assistance Publique-Hôpitaux de Paris, Département de Neurologie, Groupe Hospitalier Pitié-Salpetrière, Paris, France

2 Assistance Publique-Hôpitaux de Paris, Département de Neurophysiologie, Groupe Hospitalier Pitié-Salpêtrière, Paris, France

3 Assistance Publique-Hôpitaux de Paris, Laboratoire de Biochimie Métabolique, Groupe Hospitalier Pitié-Salpêtrière, Paris, France

4 Centre de Référence Neurométabolique Adulte, Groupe Hospitalier Pitié-Salpêtrière, Paris, France

5 Université Pierre et Marie Curie, Groupe de Recherche Clinique Neurométabolique, Paris, France
6 Sorbonne Universités, UPMC-Paris 6, UMR S 1127 and Inserm U 1127, and CNRS UMR 7225, and ICM, F-75013 Paris, France

7 Laboratoire d'imagerie biomédicale (LIB), F-75013 Paris, France

8 Bioinformatics and Biostatistics Core Facility, iCONICS, IHU-A-ICM, ICM, F-75013, Paris, France

9 CNRS-UMR 7241/INSERM U1050, CIRB, Collège de France, UPMC, Paris, France

10 Assistance Publique-Hôpitaux de Paris, Service de Neurologie, Hôpital Universitaire d'Avicenne, Bobigny, France

11 Assistance Publique-Hôpitaux de Paris, Département de Génétique, Groupe Hospitalier Pitié-Salpêtrière, Paris, France

12 Reference Center for Neurometabolic Diseases, Department of Genetics, La Pitié-Salpêtrière University Hospital, 47 Boulevard de l'Hôpital, 75013 Paris, France 
Conclusion CDCA may reverse the pathophysiological process in patients with CTX, especially if treatment is initiated early in the disease process. Besides tendon xanthoma, this study stresses the need to consider plasma cholestanol measurement in any patient with infantile chronic diarrhea and/or jaundice, juvenile cataract, learning disability and/or autism spectrum disorder, pyramidal signs, cerebellar syndrome or peripheral neuropathy.

\section{Introduction}

Cerebrotendinous xanthomatosis (CTX) is due to bi-allelic mutations in the CYP27A1 gene and results in a multisystem progressive disease (Salen and Steiner 2017). Its wide clinical spectrum mostly encompasses non-specific manifestations and overlaps with various disorders. Hence, the disease is under-diagnosed (Appadurai et al 2015, Salen and Steiner 2017) despite the existence of a simple screening test, based on the measurement of plasma cholestanol. We have recently highlighted that diarrhea almost always develops within the first year of life, cataract and school difficulties between 5 and 15 years of age, usually preceding by about a decade of motor or psychiatric symptoms (Degos et al 2016). Although some patients may have autistic features early in the disease course (Stelten et al 2017), there is a critical therapeutic window in most patients with CTX before the onset of disabling neuropsychiatric symptoms (Yahalom et al 2013, Degos et al 2016).

Mutations in the CYP27A1 gene result in the deficiency of mitochondrial cytochrome P-450 enzyme sterol 27-hydroxylase. This defect interferes with the initial side chain oxidation step of the sterol intermediates in the alternative bile acid pathway. This results in reduced synthesis of chenodeoxycholic acid (CDCA), high production of cholestanol and its subsequent accumulation in different tissues, as well as increased levels of cholesterol in plasma and bile alcohols in urine (Berginer et al 1984, Nie et al 2014). Evidence that cholestanol may be neurotoxic is supported by the finding of cholestanol deposition and apoptosis in neuronal cells, most notably Purkinje cells, in the cerebellum of rats fed a $1 \%$ cholestanol diet (Inoue et al 1999). Together with decreased levels of CDCA, sterol intermediates are shunted into the microsomal pathway for cholic acid formation (Salen et al 1991).

CDCA remains the treatment of choice in CTX as it downregulates $C Y P 7 A$, restores the imbalance between CDCA and cholic acid, and is the only drug that has shown effectiveness on neurological symptoms so far (Nie et al 2014, Salen and Steiner 2017). The exogenous supply of CDCA acts by restoring a negative feedback in the endogenous acid bile and cholestanol synthesis. This drastically lowered plasma cholestanol concentrations in patients and its accumulation in tissues (Berginer et al 1984, Nie et al 2014). CDCA nearly normalized the aberrant sterols profile found in patients with CTX (Mignarri et al 2016). While initial studies with CDCA reported clear short-term clinical benefit in most patients with CTX (Berginer et al 1984, van Heijst et al 1998), long-term studies have rather reported clinical stabilization (Mondelli et al 2001, Mignarri et al 2011, Yahalom et al 2013) and, sometimes, neurological deterioration (Pilo-de-la-Fuente et al 2011). Likewise, electrophysiological studies using transcranial magnetic stimulation or electromyoneurography (ENMG) have highlighted that the effect of therapy may depend on the extent of irreversible structural damage to axons (Mignarri et al 2011, Ginanneschi et al 2013). To our knowledge, there are only a few studies that have evaluated the effect of CDCA on quantitative brain structural metrics in CTX (Guerrera et al 2010) and none that used diffusion weighted imaging (DWI).

We therefore analyzed clinical and neurophysiological data together with brain structural parameters in a cohort of 14 patients with CTX treated with CDCA.

\section{Patients and methods}

\section{Patients}

We collected retrospectively clinical, biochemical, electrophysiological, and imaging data from 14 genetically confirmed CTX patients followed at La Pitié-Salpêtrière University Hospital between 2007 and 2017. Each patient gave a written informed consent to participate in the study. The study was approved by the local ethics committee (CPPIdF6, La Pitié-Salpêtrière University Hospital). Disease severity was scored using the Expanded Disability Status Scale (EDSS). Ataxia severity was evaluated with the Scale for the Assessment and Rating of Ataxia (SARA) score, which ranges from 0 (no cerebellar symptoms) to 40 (most severe cerebellar symptoms), in a subset of patients $(n=6)$ enrolled after 2010.

\section{Plasma cholestanol}

An UPLC-MSMS with isotopic dilution method was developed to measure plasma cholestanol as described (Marelli et al 2018). Briefly, deuterated cholestanol, used as internal standard, was mixed with plasma EDTA, after hydrolysis and extraction with $\mathrm{n}$-hexane, and free cholestanol was derivatized to picolinyl esters (Honda et al 2008). Derivatized cholestanol was detected and quantified using a triple quadripole mass spectrometer TQD coupled to an UPLC Acquity system (Waters). 


\section{Electroneuromyography}

The electrophysiological studies comprised motor and sensory conduction studies as well as needle electromyogram. We recorded (i) distal latencies and distal conduction action potentials (dCMAP) of deep peroneal nerve/extensorum digitorum brevis muscle - when not recordable, dCMAP of the tibialis anterior were also reported - and median nerve/ abductor pollicis brevis, (ii) motor conduction nerve velocities (CV) at the leg beneath the compression site for the deep peroneal nerve, when available, and at the forearm for the median nerve, (iii) F-wave for both nerves, in ms when recorded at the distal site or $\mathrm{m} / \mathrm{s}$ when recorded at the proximal site, as well as (iv) the sensory nerve action potential (SNAP) of the sural nerve (orthodromic), the superficial peroneal nerve (orthodromic), and the median nerve (antidromic at the palm). CV were considered as reduced if, in more than one nerve, they were $<48 \mathrm{~m} / \mathrm{s}$ in the upper limbs and $<42 \mathrm{~m} / \mathrm{s}$ for the lower limbs, according to our laboratory reference values.

\section{Structural brain MRI}

MRI data were acquired on a SIGNA Hdx scanner (General Electrics Medical Systems, Milwaukee, WI) with a 3 T static magnetic field and the 8 channels HR BRAIN coil. The protocol included an axial 3D T1-weighted volume $(\mathrm{TR} / \mathrm{TE} / \mathrm{TI}=$ $600 / 3.1 / 380 \mathrm{~ms}$, flip angle $=15^{\circ}$, voxel size $1 \mathrm{~mm}$ isotropic with a field of view of $256 \times 256 \mathrm{~mm}^{2}$ ) dedicated to volumetry assessments and a DWI sequence (echo-planar spin-echo) consisting of 12 volumes of isotropic diffusion gradient directions $\left(\mathrm{TR} / \mathrm{TE}=12,000 / 86 \mathrm{~ms}, \mathrm{~b}\right.$ value $=1000 \mathrm{~mm}^{2} / \mathrm{s}$, voxel size $3 \mathrm{~mm}$ isotropic with a field of view of $256 \times 256 \mathrm{~mm}^{2}$ ) with an additional non-diffusion weighted reference volume, dedicated to within-brain-tissues diffusion assessments.

3D T1-weighted volumes were analyzed using the longitudinal stream (Reuter et al 2012) of the Freesurfer image analysis suite, which is documented and freely available for download online (surfer.nmr.mgh.harvard.edu/). Briefly, this pipeline increases reliability of longitudinal measure in constraining atlas registration and brain parcellations with a within-subject template created from robust inverse consistent registration. Only the resulting volumes of subcortical segmentation were selected for further analysis.

DWI has been pre-processed with the Functional MRI of the Brain (FMRIB) software library package 5.0 (www.fmrib. ox.ac.uk/fsl/), consisting of brain extraction, correction for motion, and geometric distortions. The diffusion tensor model was then fitted to generate diffusion metrics of interest, fractional anisotropy (FA), and radial diffusivity (RD). We did not include mean diffusivity since this metric only gives information on the total amount of diffusion within a voxel but not on tissue organization or orientation.
Furthermore, the direction of the axial diffusivity does not always correspond to the underlying tissue architecture or fiber tract direction (O'Donnell and Westin 2011). For each exam, FA map was co-registered with 3D T1-weighted volume by using the efficient registration tool NyftiReg (Modat et al 2010). In NiftyReg, the deformation of FA map to T1 volume was computed by using cubic B-Spline. The final grid was $5 \times 5 \times 5 \mathrm{~mm}^{3}$. Averaged diffusion metrics were finally computed within each brain parcel identified with the Freesurfer pipeline. A mask of cerebrospinal fluid (CSF) was extracted from the mean diffusivity map by using Otsu's algorithm (Otsu 1979). In parallel, the ICBM-DTI-81 white-matter labels atlas of 48 regions of interest (Mori et al 2008) was resampled onto the $3 \mathrm{D}-\mathrm{T} 1$ space of each patient using NyftiReg. The diffusion maps resampling was achieved by a cubic interpolation onto the $\mathrm{T} 1 \mathrm{space}\left(1 \mathrm{x} 1 \mathrm{x} 1 \mathrm{~mm}^{3}\right)$. Averaged diffusion metrics were finally computed within each region of the white-matter atlas after exclusion of CSF voxels.

\section{Statistics}

Means were compared with paired t-tests or Wilcoxon signed ranked tests as deemed appropriate and correlations were evaluated with Spearman coefficient (Sp). The Holm-Bonferroni step-down method of correction was applied for multiple tests, considered significant for $p<0.05$.

\section{Results}

\section{Patient's characteristic at baseline}

General characteristics of CTX patients at baseline are summarized in Table 1. We studied three males and 11 females belonging to ten families, with a mean age at evaluation of 29 \pm 15 years (range: $8-59$ years). Eleven patients were previously described (Degos et al 2016). At baseline, plasma cholestanol was significantly elevated in all patients with a mean of $62 \pm 25 \mu \mathrm{mol} / 1$ (range: $20-98 \mu \mathrm{mol} / \mathrm{l}$ ). Ten patients presented with an infantile-onset diarrhea and 11 required surgery for a bilateral cataract. Except for four patients with no overt motor dysfunction (eTable 1 in the Supplement, P5-910-11), CTX patients presented mainly with cerebellopyramidal $(n=5)$, pyramidal $(n=3)$ or neuropathic $(n=2)$ dysfunction. All patients exhibited cognitive dysfunction, with learning difficulties and/or dysexecutive syndrome, and two patients presented with epilepsy. Three patients had osteoporosis but tendon xanthoma were clinically detected in only two patients. At baseline, patients' mean EDSS was $3.0 \pm 2.3$ (range: 0-7.5). No correlation was found between disease severity and the level of plasma cholestanol or CYP27A1 genotype. 
Table 1 Clinical characteristics of the cohort of 14 patients with CTX

\begin{tabular}{|c|c|c|}
\hline \multicolumn{3}{|l|}{ Demographic } \\
\hline Gender & Female: 11 & Male: 3 \\
\hline Familial genetics & Consanguinity: 4 & Affected sibs: 6 \\
\hline \multicolumn{3}{|l|}{ Neurological examination } \\
\hline $\begin{array}{l}\text { Age at 1st examination [median; mean } \pm \mathrm{SD} \text {; } \\
\text { range] (years) }\end{array}$ & $25.5 ; 29 \pm 15.3(8-59)$ & \\
\hline Pyramidal syndrome & $10 / 14$ & \\
\hline Cerebellar ataxia & $13 / 14$ & \\
\hline Altered proprioception & $10 / 13$ & \\
\hline Psychiatric symptoms & $6 / 14$ & \\
\hline Cognitive dysfunction & $14 / 14$ & \\
\hline Delayed-decline & $10-2 / 14$ & \\
\hline Dysexecutive (frontal) & $13 / 14$ & \\
\hline Epilepsy & $2 / 13$ & \\
\hline $\begin{array}{l}\text { Delay treatment } / 1 \text { st neurocognitive symptoms } \\
{[\text { median; mean } \pm \mathrm{SD} ; \text { range] (years) }}\end{array}$ & $15.5 ; 15.9 \pm 9.7(1-30)$ & \\
\hline Osteoporosis & $3 / 14$ & \\
\hline Tendon xanthoma & $2 / 14$ & \\
\hline Peripheral neuropathy & $11 / 13$ & \\
\hline $\begin{array}{l}\text { Cholestanol }(\mu \mathrm{mol} / \mathrm{L})[\text { mean } \pm \mathrm{SD} \text {; range }] \\
(\mathrm{N}: 2-10)\end{array}$ & $62 \pm 25(20-98)$ & \\
\hline \multicolumn{3}{|l|}{ Brain MRI/MRS } \\
\hline Atrophy & $11 / 14$ & \\
\hline Global & $4 / 14$ & \\
\hline Cerebellar & $8 / 14$ & \\
\hline Periventricular T2 hypersignal & $13 / 14$ & \\
\hline Increased choline (MRS) & $12 / 13$ & \\
\hline Dentate nuclei T2 hypersignal & $14 / 14$ & \\
\hline
\end{tabular}

\section{Biochemical and clinical evolution under treatment}

Plasma cholestanol levels normalized under treatment with CDCA within a few months $(9 \pm 4 \mu \mathrm{mol} / 1$, range: $2-16$ $\mu \mathrm{mol} / 1)(p<0.001)$. Tolerance to the drug was always good, including normal liver functions. However, we observed a relapse (increase) of plasma cholestanol levels in three patients (P1-4-7) linked to poor compliance with CDCA medication.

Mean clinical follow-up under CDCA was 5 years (range: 2-9 years). Patients' mean EDSS remained roughly stable at $3.4 \pm 2.7$ (range: $0-8$ ). More specifically, EDSS worsened in three patients (Fig. 1, P2-3-8), improved in three patients (Fig. 1, P10-12-13), and remained stable in six patients (Fig. 1 and eTable 1 in the Supplement). Of note, the three patients whose disease worsened experienced the longest time between treatment start and the occurrence of the first cognitive and/or motor symptoms - respectively 32,28 , and 25 years (eTable 1 in the Supplement). When performed $(n=6)$, SARA scores improved significantly from $5.3 \pm 3.9$ (range: $1.5-12)$ to $2.0 \pm 1.4$ (range: $1-4)(p=0.043)$, in keeping with the improvement of the EDSS in the same patients - from 2.5 \pm 1.9 to $1.9 \pm 1.6(p=0.034)$ (Fig. 1 and eTable 1 in the Supplement). Patient P12, whose EDSS and SARA score improved with CDCA, also exhibited a marked improvement of her psychotic symptoms, e.g., disappearance of hallucinations and reduction in the number of anti-psychotic drugs from five to one.

\section{Electroneuromyography}

ENMG before treatment start was available for all patients except P8. Patient P8 had an ENMG after receiving CDCA for 9 months, which was normal. A length-dependent peripheral neuropathy was observed in 11 patients - all patients except P1 and P14, Table 2. Eight patients (P2-3-4-5-7-910-13) out of the 11 neuropathic patients (73\%) presented with reduced motor $\mathrm{CV}$ in at least two nerves. Distal latencies and F-waves are presented in eTable 2 in the Supplement.

In the 11 neuropathic patients treated with CDCA, dCMAP of deep peroneal nerve/extensor digitorum brevis significantly increased from a mean of $2.49 \pm 1.65 \mathrm{~m} / \mathrm{s}$ to a mean of $3.57 \pm$ $2.55 \mathrm{~m} / \mathrm{s}(p=0.015)$ (Table 2). Furthermore, motor CV in the lower limbs of patients with reduced $\mathrm{CV}$ at baseline 

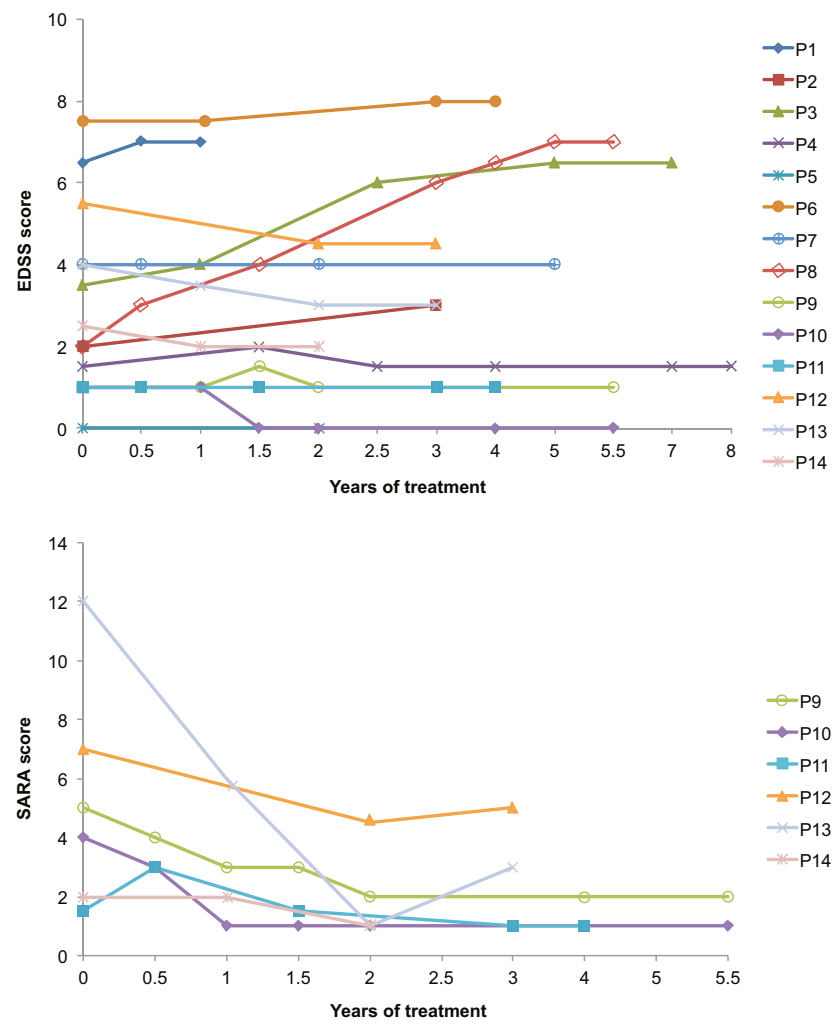

Fig. 1 Evolution of the Expanded Disability Status Scale (EDSS) and Scale for the Assessment and Rating of Ataxia (SARA) in patients with CTX treated with CDCA

significantly increased from a mean of $33.8 \pm 4.0 \mathrm{~m} / \mathrm{s}$ to a mean of $41.8 \pm 4.2 \mathrm{~m} / \mathrm{s}(p=0.002)$ (Table 2). Notably, two patients, $\mathrm{P} 4$ and $\mathrm{P} 9$, doubled their lower limbs SNAPs (Table 2).

\section{Quantitative brain MRI}

All MRI were acquired during routine clinical follow-up. This resulted at times in a loss of quality, precision, and homogeneity compared to research MRI, as previously reported. All images were visually and manually checked and not included if quality was inappropriate: (i) only axial or non-3D images making segmentation not possible or unreliable; (ii) images with severe motion artifact that made them too poor to evaluate. Before treatment, volumetric and DWI data were available for six and eight patients respectively. After three years of treatment with CDCA, volumetric and DWI data were available for four patients.

Volumetric analyses showed no overt volume loss in patients with CTX under treatment with CDCA. Moreover, DWI analyses showed improved FA of the ponto-cerebellar tract $[0.27804( \pm 0.02618,0.24101-0.31942)$ versus $0.37104( \pm$ $0.07329,0.30046-0.46674), p=0.012]$ (Fig. 2) and improved $\mathrm{RD}$ of the internal capsule $[0.00423( \pm 0.00028,0.00389-$
$0.00479)$ versus $0.00392( \pm 0.00022,0.00368-0.00411)$, $p=0.024$ ] (Fig. 2) after three years of treatment with CDCA.

We hypothesized that structural changes on the nervous system of patients treated by CDCA were more likely to occur and be detected with methods related to myelin metrics, rather than axonal ones. Likewise, the improvement on electrophysiological parameters that we observed may reflect a benefit to the peripheral myelin, whereas the improvement on brain diffusion metrics may reflect a benefit to the central myelin. Hence, we studied the relationship between peripheral and central myelin metrics. We observed significant correlations between lower limbs dCMAP and FA metrics, especially the corpus callosum $(\operatorname{Sp~} 0.729 p=0.020)$ and the internal capsule (Sp 0.693, $p=0.036$ ) (Fig. 3). Significant correlations were also found between lower limbs dCMAP and RD metrics: the overall $\mathrm{RD}$ ( $\mathrm{Sp}-0.831, p<0.001)$, the cingulum ( $\mathrm{Sp}-0.891$, $p<0.001)$, the corpus callosum ( $\mathrm{Sp}-0.814, p=0.004)$, the corona radiata $(\mathrm{Sp}-0.823, p=0.004)$, the internal capsule $(\mathrm{Sp}$ $-0.790, p=0.004)$, the posterior thalamic radiations $(\mathrm{Sp}-0 /$ $787, p=0.004)$, and the external capsule $(\mathrm{Sp}-0.748, p=$ 0.003) (Fig. 3).

\section{Discussion}

In our cohort of 14 patients with CTX, CDCA either improved or stabilized motor functions when treatment was initiated less than 25 years after the onset of neuropsychiatric manifestations. Moreover, CDCA was able to significantly improve electrophysiological parameters of neuropathic patients. Brain structural analyses showed a global stabilization of volumetric parameters, and improvement of FA and RD in some brain regions under treatment. We observed no hepatic toxicity under CDCA.

It was postulated that, after significant neurological pathology has developed in CTX, the effect of treatment with CDCA is limited and patients ought to continue deteriorating (Yahalom et al 2013). Here, we provide further evidence that patients with CTX whose treatment was initiated more than 25 years after the onset of neurological symptoms continued their clinical deterioration, despite improved ENMG metrics in some of them. However, unlike most studies in adult patients with CTX leaning toward long-term clinical stabilization under CDCA (Mondelli et al 2001, Mignarri et al 2011, Yahalom et al 2013), we observed a significant clinical improvement in patients up to 25 years old, whose treatment was initiated less than 15 years after the onset of neurological symptoms. Furthermore, CDCA was associated with improved nerve conduction studies, but stable amplitudes, in 20 patients (Ginanneschi et al 2013). Here, we showed that CDCA can significantly increase nerve conduction in patients with reduced CV, as well as improve dCMAP in the lower limbs. 
Table 2 ENMG parameters of the 14 CTX patients at (or just before) treatment (M0), at M12 (or the next available ENMG), and at the last follow-up

\begin{tabular}{|c|c|c|c|c|c|c|c|c|}
\hline \multirow[t]{3}{*}{ Patient } & \multirow[t]{3}{*}{ Time } & \multicolumn{4}{|l|}{ Motor study } & \multirow{2}{*}{\multicolumn{3}{|c|}{$\begin{array}{l}\text { Sensory study } \\
\text { SNAP }\end{array}$}} \\
\hline & & \multicolumn{2}{|l|}{ Median } & \multicolumn{2}{|l|}{ Deep peroneal } & & & \\
\hline & & dCMAP (mV) & $\mathrm{CV}(\mathrm{m} / \mathrm{s})$ & dCMAP (mV) & $\mathrm{CV}(\mathrm{m} / \mathrm{s})$ & Sural $(\mu \mathrm{V})$ & $\mathrm{SP}(\mu \mathrm{V})$ & Median $(\mu \mathrm{V})$ \\
\hline \multirow[t]{2}{*}{$\mathrm{P} 1$} & M0 & 5.4 & 42 & 3.4 & 52 & - & - & - \\
\hline & M29 & 7.8 & 56 & 5.8 & - & 56 & 50 & 46 \\
\hline \multirow[t]{3}{*}{$\mathrm{P} 2$} & M0 & 11.2 & 40 & 2.4 & 28 & 7 & 4 & 130 \\
\hline & M12 & 11.5 & 45 & 3.5 & 34 & 8 & 5 & 85 \\
\hline & M39 & 10.1 & 44 & 5.0 & 36 & - & - & - \\
\hline \multirow[t]{3}{*}{ P3 } & M0 & 10.0 & 54 & 2.5 & 39 & 16 & 18 & - \\
\hline & M12 & 8.2 & 49 & 2.8 & 42 & 11 & 17 & - \\
\hline & M108 & 6.2 & - & 2.8 & 45 & 13 & - & 55 \\
\hline \multirow[t]{3}{*}{ P4 } & M0 & 5.2 & 37 & $\mathrm{NO} / 3.3^{*}$ & $-/ 40 *$ & 15 & 11 & 59 \\
\hline & M18 & 6.8 & 42 & $\mathrm{NO} / 3.5^{*}$ & $-156^{*}$ & 13 & - & 21 \\
\hline & M98 & 8.5 & 47 & $0.2 / 4.5^{*}$ & $34 / 44 *$ & 31 & - & 73 \\
\hline \multirow[t]{3}{*}{ P5 } & M0 & 9.7 & 49 & 3.6 & 35 & 33 & 21 & - \\
\hline & M30 & 9.7 & 52 & 5.0 & 42 & 69 & 17 & 88 \\
\hline & M60 & 9.8 & 60 & 6.7 & 46 & 31 & 13 & 191 \\
\hline \multirow[t]{3}{*}{ P6 } & M0 & 9.1 & 50 & $0.4 / 1.4 *$ & $35 / 63 *$ & NO & NO & 8 \\
\hline & M12 & 13.0 & 49 & $\mathrm{NO} / 0.9^{*}$ & $-1-$ & NO & NO & 70 \\
\hline & M48 & 13.8 & - & $\mathrm{NO} / 2.5^{*}$ & $-/ 65^{*}$ & $\mathrm{NO}$ & NO & 32 \\
\hline \multirow[t]{3}{*}{ P7 } & M0 & 4.7 & - & $\mathrm{NO} / 3.2 *$ & $-/ 34^{*}$ & 12 & 6 & 17 \\
\hline & M6 & 4.8 & 33 & $\mathrm{NO} / 5.2 *$ & $-/ 40 *$ & 16 & 4 & - \\
\hline & M39 & 5.5 & 39 & $\mathrm{NO} / 7.1^{*}$ & $-/ 34 *$ & 14 & 6 & 33 \\
\hline \multirow[t]{3}{*}{ P8 } & M0 & - & - & - & - & - & - & - \\
\hline & M9 & 12.9 & 50 & 6.4 & 40 & - & 24 & 102 \\
\hline & M36 & 12.7 & - & 6.3 & 44 & - & 23 & - \\
\hline \multirow[t]{3}{*}{ P9 } & M0 & 10.9 & 43 & 3.2 & 34 & 27 & 17.9 & 101 \\
\hline & M12 & 12.2 & 45 & 3.8 & 36 & 24 & 15 & 53 \\
\hline & M48 & 13.8 & 48 & 3.2 & 39 & 52 & 14 & 130 \\
\hline \multirow[t]{3}{*}{ P10 } & M0 & 10.3 & 48 & 3.0 & 33 & 42 & 25 & 70 \\
\hline & M12 & 10.2 & 50 & 4.2 & 40 & 18 & 14 & 34 \\
\hline & M36 & 14.5 & 54 & 4.4 & 43 & 61 & - & 103 \\
\hline \multirow[t]{3}{*}{ P11 } & M0 & 8.8 & 58 & 4.4 & 48 & 44 & 16 & 59 \\
\hline & M18 & 6.1 & 56. & 4.1 & 51 & 24 & 10 & 44 \\
\hline & M30 & 11.8 & 57 & 6.6 & 45 & 33 & 17 & 44 \\
\hline \multirow[t]{3}{*}{$\mathrm{P} 12$} & M0 & 6.7 & 58 & 4.5 & 45 & 21 & 7 & 75 \\
\hline & M21 & 9.7 & 66 & 5.3 & 48 & 20 & 16 & 153 \\
\hline & M33 & 9.2 & 55 & 5.1 & 51 & 17 & 12 & 102 \\
\hline \multirow[t]{3}{*}{ P13 } & M0 & 11.2 & 44 & 3.4 & 33 & 27 & 14 & 164 \\
\hline & M10 & 7.0 & 50 & 4.2 & 38 & 30 & - & 143 \\
\hline & M20 & 9.3 & 66 & 5.3 & 48 & 20 & 16 & 78 \\
\hline \multirow[t]{3}{*}{ P14 } & M0 & 10.5 & 53 & 5.8 & 41 & 37 & 32 & 89 \\
\hline & M12 & 11.5 & 52 & 8.9 & 41 & 43 & 40 & 88 \\
\hline & M24 & 10.9 & 51 & 7.9 & 41 & 30 & 30 & - \\
\hline
\end{tabular}

$\mathrm{SNAP}=$ sensory nerve action potential; $\mathrm{dCMAP}=$ distal compound muscle action potential; $\mathrm{CV}=$ conduction velocity; $\mathrm{SP}=$ superficial peroneal; - = not available; $\mathrm{NO}=$ not obtainable; * for the deep peroneal, at the extensor digitorum brevis when not recordable at the tibialis anterior 
Fig. 2 Diffusion weighted imaging analyses showed improved fractional anisotropy of the ponto-cerebellar tract (red) and improved radial diffusivity of the internal capsule (yellow) with CDCA
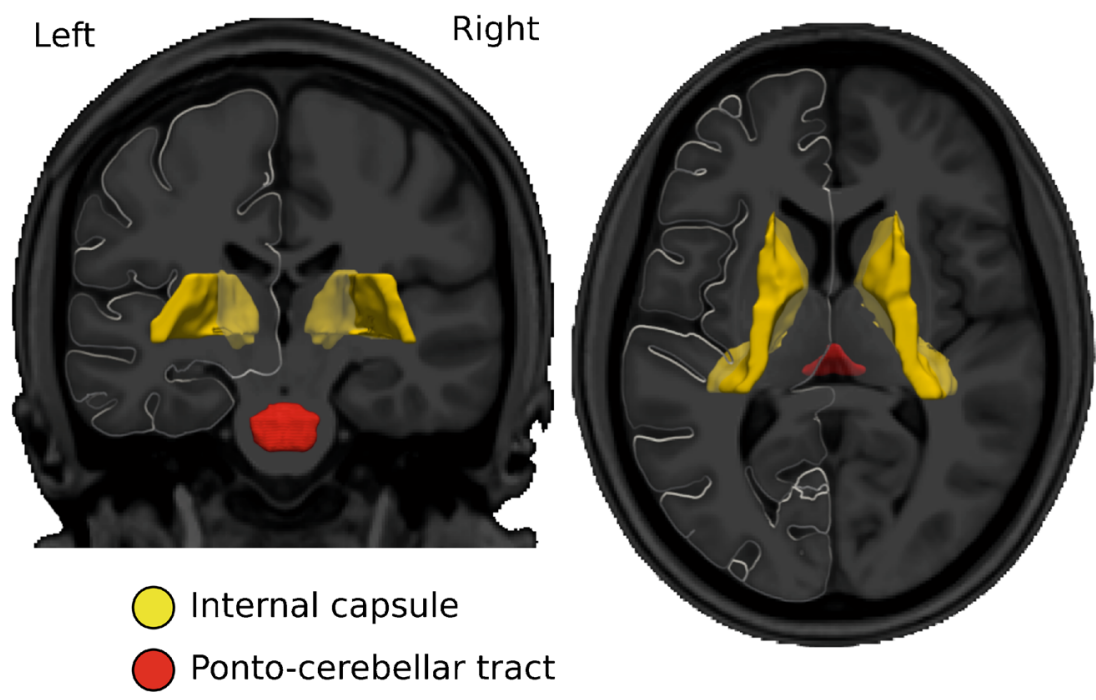

Since 1994, brain MRI has been recognized to be useful for the diagnosis of CTX, especially white matter changes in the dentate nuclei (Barkhof et al 2000), but of little value for treatment evaluation (Berginer et al 1994). More recently, the presence of cerebellar vacuolation images has been associated with a poor response to treatment, and therefore considered as a prognostic marker (Mignarri et al 2017). Few quantitative structural MRI studies have been conducted in
Fig. 3 Correlation between electromyoneurography and diffusion metrics. There were significant correlations between distal compound muscle action potential of the deep peroneal (AmpDP) and fractional anisotropy (FA) in the corpus callosum and the internal capsule (red).

Significant correlations were also found between AmpDP and radial diffusivity (RD) in the cingulum, the corpus callosum, the corona radiate, the internal capsule, the posterior thalamic radiations, and the external capsule (blue)
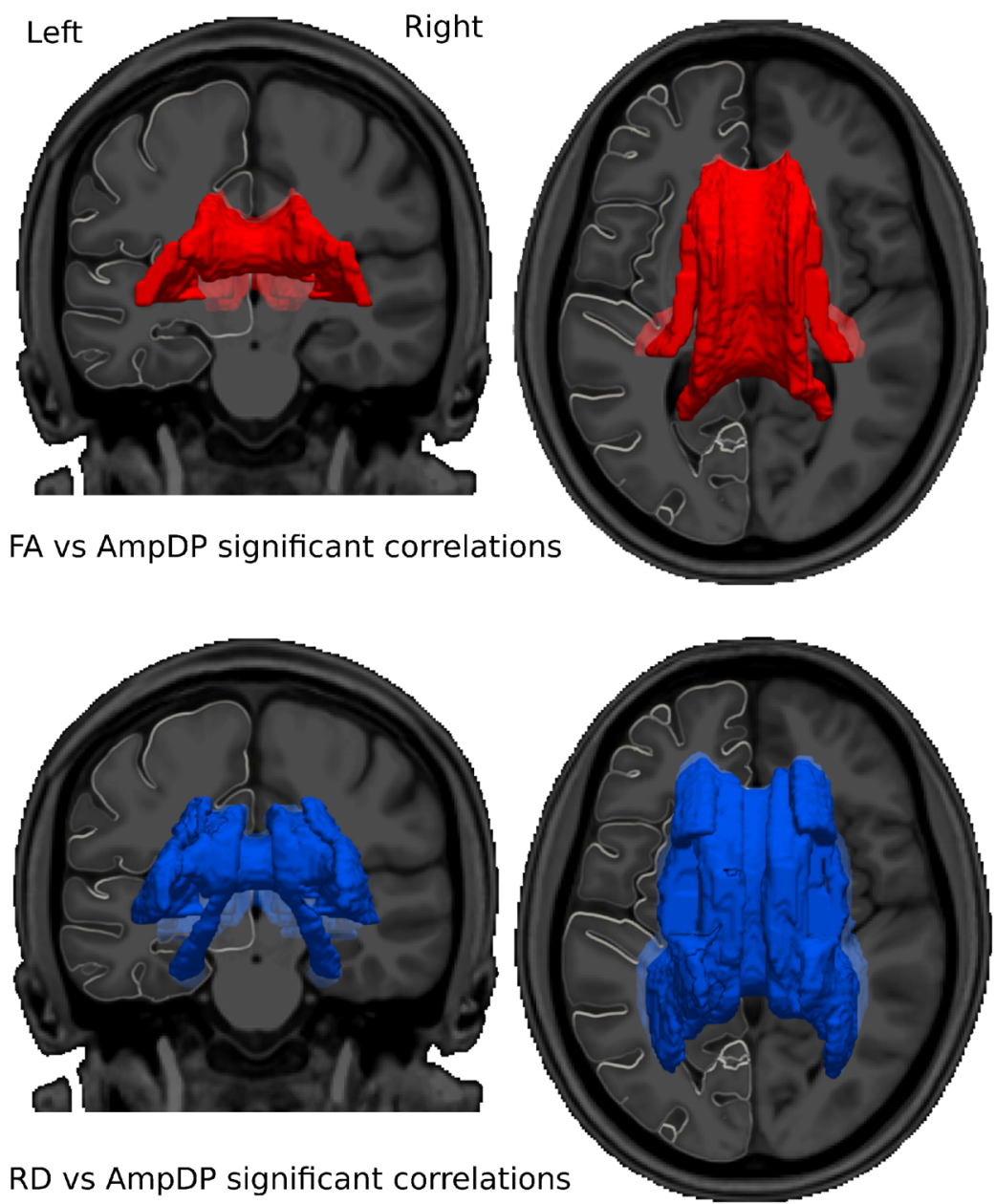
CTX. In untreated patients with CTX, brain structural changes correlated with clinical parameters (Inglese et al 2003, Chang et al 2010). One volumetric study showed higher rates of atrophy in patients with CTX treated with CDCA than controls but no data was available in untreated patients (Guerrera et al 2010). Here, we showed in a subset of patients with CTX that CDCA can prevent brain volume loss and improve fiber integrity of the ponto-cerebellar tract and the internal capsule. Since RD is a marker of myelin homeostasis, the greater correlation between ENMG metrics and RD might suggests a similar effect of CDCA treatment on both peripheral and central myelin. This study also emphasizes that diffusion metrics may be more sensitive to change than volumetry, as reported in other neurometabolic diseases (Masingue et al 2017).

In conclusion, the possibility to reverse the pathophysiological process in patients with CTX stresses that the disease must be diagnosed as early as possible. Accordingly, a suspicion index has been proposed (Mignarri et al 2014). All the more, besides tendon xanthoma, we advocate to measure plasma cholestanol in all patients presenting with any of the following clinical presentation: infantile chronic diarrhea and/or jaundice, juvenile cataract, learning disability and/or autism spectrum disorder, pyramidal signs, cerebellar syndrome or peripheral neuropathy.

Acknowledgements We are very grateful to the patients who participated in this study. We would also like to thank Damien Galanaud for MRI methods development, Isaac Adanyeguh for technical assistance, Philippe Couvert for molecular analyses, Frédéric Sedel and Yann Nadjar for patients referral. This study was supported the Investissements d'Avenir (Paris Institute of Neurosciences - IHU) grant number ANR-10-IAIHU-06.

\section{Compliance with ethical standards}

Conflict of interest Maria del Mar Amador, Marion Masingue, Rabab Debs, Foudil Lamari, Vincent Perlbarg, Emmanuel Roze, Bertrand Degos declare that they have no conflict of interest.

Fanny Mochel has received an education grant from Sigma Tau pharmaceuticals.

Animal rights This article does not contain any studies with animal subjects performed by the any of the authors.

\section{References}

Appadurai V, DeBarber A, Chiang PW et al (2015) Apparent underdiagnosis of cerebrotendinous xanthomatosis revealed by analysis of $\sim 60,000$ human exomes. Mol Genet Metab 116:298-304

Barkhof F, Verrips A, Wesseling P et al (2000) Cerebrotendinous xanthomatosis: the spectrum of imaging findings and the correlation with neuropathologic findings. Radiology 217:869-876

Berginer VM, Salen G, Shefer S (1984) Long-term treatment of cerebrotendinous xanthomatosis with chenodeoxycholic acid. N Engl J Med 311:1649-1652
Berginer VM, Berginer J, Korczyn AD, Tadmor R (1994) Magnetic resonance imaging in cerebrotendinous xanthomatosis: a prospective clinical and neuroradiological study. J Neurol Sci 122:102-108

Chang CC, Lui CC, Wang JJ et al (2010) Multi-parametric neuroimaging evaluation of cerebrotendinous xanthomatosis and its correlation with neuropsychological presentations. BMC Neurol 10:59-66

Degos B, Nadjar Y, Amador Mdel M et al (2016) Natural history of cerebrotendinous xanthomatosis: a paediatric disease diagnosed in adulthood. Orphanet J Rare Dis 11:41-44

Ginanneschi F, Mignarri A, Mondelli M et al (2013) Polyneuropathy in cerebrotendinous xanthomatosis and response to treatment with chenodeoxycholic acid. J Neurol 260:268-274

Guerrera S, Stromillo ML, Mignarri A et al (2010) Clinical relevance of brain volume changes in patients with cerebrotendinous xanthomatosis. J Neurol Neurosurg Psychiatry 81:1189-1193

Honda A, Yamashita K, Miyazaki H et al (2008) Highly sensitive analysis of sterol profiles in human serum by LC-ESI-MS/MS. J Lipid Res 49:2063-2073

Inglese M, De Stefano N, Pagani E et al (2003) Quantification of brain damage in cerebrotendinous xanthomatosis with magnetization transfer MR imaging. Am J Neuroradiol 24:495-500

Inoue K, Kubota S, Seyama Y (1999) Cholestanol induces apoptosis of cerebellar neuronal cells. Biochem Biophys Res Commun 256:198_203

Marelli C, Lamari F, Rainteau D et al (2018) Plasma oxysterols: biomarkers for diagnosis and treatment in spastic paraplegia type 5 . Brain 141:72-84

Masingue M, Adanyeguh I, Nadjar Y et al (2017) Evolution of structural neuroimaging biomarkers in a series of adult patients with Niemannpick type C under treatment. Orphanet J Rare Dis 12:22-28

Mignarri A, Rossi S, Ballerini M et al (2011) Clinical relevance and neurophysiological correlates of spasticity in cerebrotendinous xanthomatosis. J Neurol 258:783-790

Mignarri A, Gallus GN, Dotti MT, Federico A (2014) A suspicion index for early diagnosis and treatment of cerebrotendinous xanthomatosis. J Inherit Metab Dis 37:421-429

Mignarri A, Magni A, Del Puppo M et al (2016) Evaluation of cholesterol metabolism in cerebrotendinous xanthomatosis. J Inherit Metab Dis 39:75-83

Mignarri A, Dotti MT, Federico A et al (2017) The spectrum of magnetic resonance findings in cerebrotendinous xanthomatosis: redefinition and evidence of new markers of disease progression. J Neurol 264: 862-874

Modat M, Ridgway GR, Taylor ZA et al (2010) Fast free-form deformation using graphics processing units. Comput Methods Prog Biomed 98:278-284

Mondelli M, Sicurelli F, Scarpini C, Dotti MT, Federico A (2001) Cerebrotendinous xanthomatosis: 11-year treatment with chenodeoxycholic acid in five patients. An electrophysiological study. J Neurol Sci 190:29-33

Mori S, Oishi K, Jiang H et al (2008) Stereotaxic white matter atlas based on diffusion tensor imaging in an ICBM template. NeuroImage 40: $570-582$

Nie S, Chen G, Cao X, Zhang Y (2014) Cerebrotendinous xanthomatosis: a comprehensive review of pathogenesis, clinical manifestations, diagnosis, and management. Orphanet J Rare Dis 9:179-189

O'Donnell LJ, Westin CF (2011) An introduction to diffusion tensor image analysis. Neurosurg Clin N Am 22(2):185-196

Otsu N (1979) A threshold selection method from gray-level histograms. IEEE Trans Sys Man Cyber 9:62-66

Pilo-de-la-Fuente B, Jimenez-Escrig A, Lorenzo JR et al (2011) Cerebrotendinous xanthomatosis in Spain: clinical, prognostic, and genetic survey. Eur J Neurol 18:1203-1211

Reuter M, Schmansky NJ, Rosas HD, Fischl B (2012) Within-subject template estimation for unbiased longitudinal image analysis. NeuroImage 61:1402-1418 
Salen G, Shefer S, Berginer V (1991) Biochemical abnormalities in cerebrotendinous xanthomatosis. Dev Neurosci 13:363-370

Salen G, Steiner RD (2017) Epidemiology, diagnosis, and treatment of cerebrotendinous xanthomatosis (CTX). J Inherit Metab Dis 40: 771-781

Stelten BML, Bonnot O, Huidekoper HH, et al (2017) Autism spectrum disorder: an early and frequent feature in cerebrotendinous xanthomatosis. J Inherit Metab Dis doi:10.1007/s10545-017-0086-7 van Heijst AF, Verrips A, Wevers RA et al (1998) Treatment and followup of children with cerebrotendinous xanthomatosis. Eur J Pediatr 157:313-316

Yahalom G, Tsabari R, Molshatzki N et al (2013) Neurological outcome in cerebrotendinous xanthomatosis treated with chenodeoxycholic acid: early versus late diagnosis. Clin Neuropharmacol 36:78-83 